\title{
Thermoelectric properties of $\mathrm{GaAs} / \mathrm{Ga}_{1-x} \mathrm{Al}_{x} \mathrm{As}$ heterojunctions in the fractional quantum Hall regime
}

\author{
U. Zeitler ${ }^{*, a, 1}$, R. Fletcher ${ }^{b}$, J.C. Maan ${ }^{\text {a,c }}$, C.T. Foxon ${ }^{\text {d }}$, J.J. Harris ${ }^{\text {e }}$, P. Wyder ${ }^{a}$ \\ ${ }^{a}$ High Magnetic Field Laboratory (MPIF-CNRS), B.P. 166, F-38042, Grenoble Cedex 9, France \\ ${ }^{b}$ Physics Department, Queen's University, Kingston, Ontario, Canada K7L $3 N 6$ \\ ' High Field Magnet Laboratory, University of Nijmegen, 6525 ED Nijmegen, The Netherlands \\ ${ }^{d}$ Department of Physics, University of Nottingham, Nottingham NG7 2RD, UK \\ ${ }^{e}$ Semiconductor Interdisciplinary Research Centre, Imperial College, London SW7 2BZ, UK
}

(Received 21 April 1993; accepted for publication 11 August 1993)

\begin{abstract}
We present experimental data on the thermoelectric power of a two-dimensional clectron gas in the fractional quantum Hall regime. The thermopower is shown to be due to phonon-drag and therefore reflects the coupling of phonons to the electronic quasiparticle states. The general behaviour of the thermoelectric power as a function of applied magnetic field and temperature is compared to recent theoretical predictions of the electron-phonon drag in quantizing magnetic fields.
\end{abstract}

The thermoelectric power (TEP) of two-dimensional electron gases (2DEG) in GaAs/ $\mathrm{Ga}_{1-x} \mathrm{Al}_{x}$ As heterojunctions mainly reflects the coupling of the phonons to the electronic states, as opposed to resistivity measurements which are sensitive to the electron-impurity scattering. TEP measurements therefore provide an important complementary tool for a better understanding of the electronic states in a 2DEG. At high magnetic fields, where only the lowest Landau level is occupied, and at low temperature, where the fractional quantum Hall (FQH) states develop, the nature of the phonon coupling to the electronic quasiparticles is an especially interesting

\footnotetext{
* Corresponding author.

${ }^{1}$ Present address: High Field Magnet Laboratory, University of Nijmegen, $6525 \mathrm{ED}$, The Netherlands.
}

problem. However, only experimental TEP data in the integer quantum Hall regime have been reported previously $[1,2]$.

The aim of our experiments was the investigation of the TEP of a $2 \mathrm{DEG}$ in the FQH regime. We will present the overall behaviour of the TEP in this region and will show that its variation as a function of magnetic field and temperature agrees qualitatively with a recent theory of the electron-phonon drag effect in an ideally pure $2 \mathrm{DEG}$ [3]. Howcver, an cxperimentally observed strong decrease of the TEP in very high magnetic fields (filling factors below $1 / 5$ ) cannot yet be explained.

The thermoelectric power $S$ of an electron system is due to an applied temperature gradient $\boldsymbol{\nabla} T$ which leads to a thermal current $j_{\text {th }}=\boldsymbol{\epsilon} \boldsymbol{\nabla} T$ ( $\epsilon$ is the thermoelcctric tcnsor). In the steady state, where no total current is flowing, an oppo- 
site current $j_{\mathrm{el}}=\sigma \boldsymbol{E}$ ( $\sigma$ is the conductivity tensor) has to exist and an electric field is generated $\boldsymbol{E}=\mathrm{S} \boldsymbol{\nabla} T$ to produce this, leading to the relation: $\mathrm{S}=\sigma^{-1} \epsilon$. For high mobility samples and at high fields, where $\rho_{x x} \ll \rho_{x y}$, the two independent components of the TEP in a $2 \mathrm{DEG}$ resulting from this equation can be simplified to $S_{x x} \approx$ $-\epsilon_{y x} \rho_{y x}$ (within $2 \%$ for our samples) and $S_{y x} \approx$ $\epsilon_{x x} \rho_{y x}$ (within $10 \%$ ).

We have investigated the thermoelectric properties of three different MBE-grown high mobility samples (kindly provided by Philips Research Laboratories, Redhill, UK; labeled G645, G647 and G650 with electron concentrations of 0.86 , 0.43 , and $1.0 \times 10^{15} \mathrm{~m}^{-3}$ and mobilities of 230 , 270 , and $190 \mathrm{~m}^{2} / \mathrm{V} \cdot \mathrm{s}$ ). The 2DEG were grown on a semi-insulating GaAs substrate with dimensions of about $10 \times 3 \times 0.5 \mathrm{~mm}^{3}$.

In order to perform TEP measurements, each sample was mounted freestanding inside a vacuum. One end was thermally anchored by soldering it with indium to a copper arm which was in thermal contact with a ${ }^{3} \mathrm{He}$ bath, and a strain gauge heater was glued to the other end. By passing a DC current through the heater, a thermal gradient was established in the sample. We measured the temperature $T$ and the temperature gradient $\nabla T$ with two calibrated thermometers glued on the rear side of the substrate. Given the known heating power, the thermal conductivity $\lambda$ of the samples could be calculated. In order to check the thermometry in a magnetic field we have measured $\lambda$ at various fields; we did not observe any significant change as a function of the field.

The thermal conductivity of sample G647 at zero magnetic field is shown in the insert of Fig. 1. For all three samples the measured heat conductivities behave in a quantitatively similar way and are found to be close to the theoretically expected $T^{3}$ dependence in the boundary scattering limit. The slight deviation from $T^{3}$ is consistent with earlier published data [1].

The thermopower $S_{x x}$ and the Nernst-Ettingshausen coefficient $S_{y x}$ were measured with a phase-sensitive lock-in technique by applying an AC heating at $12 \mathrm{~Hz}$. We verified that the thermal response of the system was fast enough to

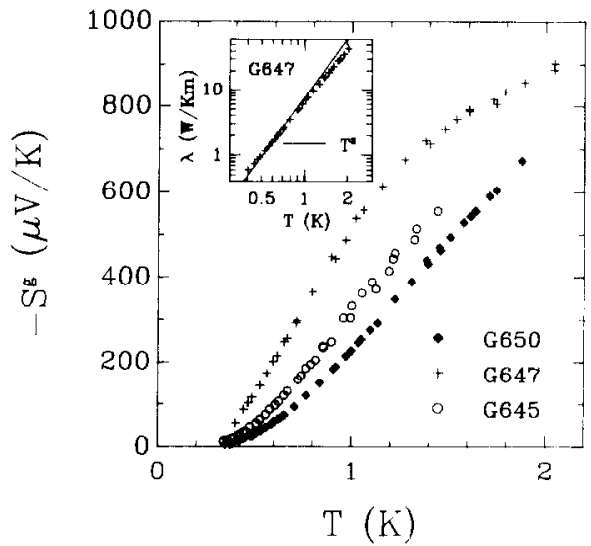

Fig. 1. The phonon-drag thermopower at zero magnetic field for three different samples. The insert shows the measured heat conductivity of the GaAs substrate of G647.

follow this frequency by obtaining the same results for $5-30 \mathrm{~Hz}$, as well as with a DC method.

Generally the TEP is the sum of two contributions: the diffusion of the charge carriers, and the drag of these carriers by phonons. At zero magnetic field the diffusion contribution to the TEP can be written as $S^{\mathrm{d}}=-\left(\pi^{2} k / 3 e\right)(p+1)\left(T / T_{\mathrm{F}}\right)$ ( $T_{\mathrm{F}}$ being the Fermi temperature and $p \approx 1$ expressing the energy dependence of the electron scattering) [4]. For our samples at $1 \mathrm{~K}$ we expect $S^{\mathrm{d}}$ to have a magnitude between 14 and $30 \mu \mathrm{V} / \mathrm{K}$ (taking $p=1$ ). The experimental magnitudes are at least an order of magnitude higher and the temperature dependence is much faster than linear, showing that the phonon drag TEP is the dominant contribution in the temperature range explored. We believe that this remains true in a magnetic field (though little is known about the diffusion component in the $\mathrm{FQH}$ regime) and so the TEP will continue to reflect the coupling of the phonons to the electronic quasiparticle states.

The zero field phonon drag TFP $S^{\mathrm{g}}$ (i.e the experimentally measured TEP after subtracting the above described diffusion contribution using $p=1$ ) is plotted in Fig. 1. In the simplest model, the phonon-drag TEP is expected to vary as $T^{3}$ [5] (which gives references to more detailed treatments; see also Refs. [6,7]). At higher temperatures the TEP begins to saturate because phonons with $k_{\|}>2 k_{\mathrm{F}}$ can no longer couple to the $2 \mathrm{DEG}$ 
( $k_{\|}$is the component of the phonon wave vector in the plane of the 2DEG, and $k_{\mathrm{F}}$ is the Fermi wave vector of the two-dimensional electrons).

In Fig. 2 we show the measured quantities $S_{x x}$ and $S_{\mathrm{yx}}$ in a magnetic field. At lower fields, i.e. in the integer quantum Hall region, the TEP $S_{x x}$ as well as the Nernst-Ettingshausen coefficient $S_{y x}$ show an oscillatory behaviour, the latter resembling the derivative of the former with respect to magnetic field. This behaviour has been reported for many different samples [1,7]. In this region standard theory explains the occurrence as well as the magnitude of the maxima in $S_{x x}$ [8], whereas $S_{y x}$ is expected to be zero. A recent theory which relates the thermoelectric tensor to the derivative of the conductivity with respect to filling factor [9] can explain the oscillations of $S_{y x}$ and its proportionality to the derivative of $S_{x x}$ with respect to magnetic field.

At higher magnetic fields, when the FQH effect becomes visible, several interesting features appear in the TEP. Some have been reported

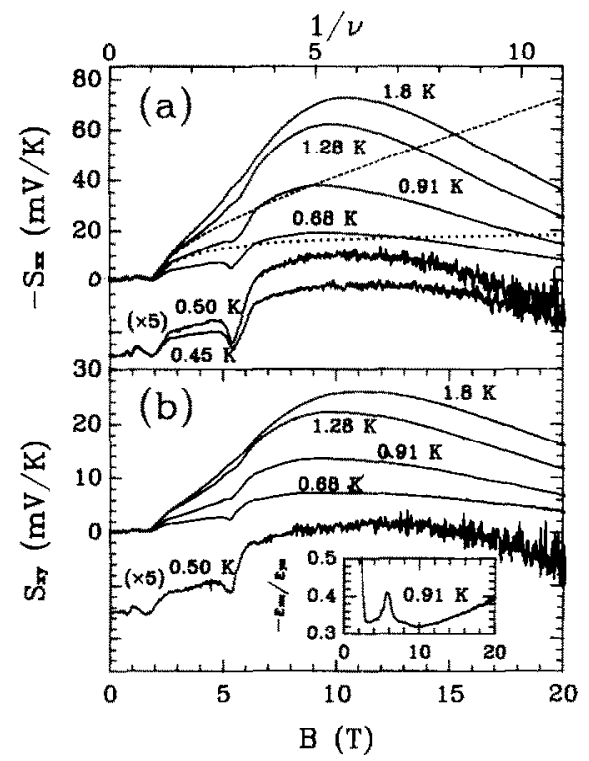

Fig. 2. (a) The TEP $S_{x x}$ of G647 for different temperatures; the dotted line represents schematically the theoretical variation with only polar interaction; the broken line includes the deformation potential. (b) The Nernst-Ettingshausen coefficient $S_{x y}$ of $\mathrm{G} 647$ for different temperatures with the ratio $j_{x} / j_{y}$ of the thermally induced currents in the insert.

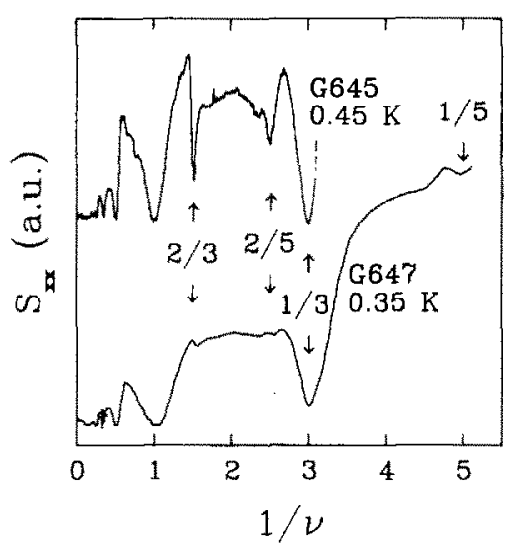

Fig. 3. The low temperature TEP $S_{x x}$ for two samples showing several well pronounced fractions.

elsewhere [7], and we shall restrict ourselves here to a brief outline. Fractional filling factors (mainly $1 / 3$ and $2 / 3$, and below $0.5 \mathrm{~K}$ also $1 / 5$ and $2 / 5$ ) are visible in the thermopower $S_{x x}$ and the Nernst-Ettingshausen coefficient $S_{y x}$ (Fig. 3), roughly comparable to their visibility in the resistivities $\rho_{x x}$ and $\rho_{x y}$ at similar temperatures. This means that the phonons cannot couple to the fractional states, but only to the thermally excited quasiparticles. However, apart from the minima, the TEP in the FQH regime is found to behave differently from the integer regime and also differently as compared to resistivity. For example, in the FQH regime the Nernst-Ettingshausen coefficient $S_{y x}$ no longer resembles the field derivative of $S_{x x}$, as is the case in the integer quantum Hall regime, and in particular does not change sign when $S_{x x}$ crosses fractional minima.

Moreover $S_{y x}$ is found to be unexpectedly large, whereas for high mobility samples we expect it to be close to zero, which might be explained as follows. Due to the Lorentz force a phonon flux in the $x$-direction induces a thermal electron current turned by the Hall angle, i.e. for $\rho_{x x} \ll \rho_{x y}$ perpendicular to it. This current must be compensated by a counter current due to an electric field which is again perpendicular to its resulting current. Therefore the electric field points in the direction of the thermal gradient. For a Hall angle $\Theta$ slightly below $90^{\circ}$, the thermally induced current should be turned by about 
$90^{\circ}-\Theta$ with respect to the perpendicular direction to the thermal gradient which corresponds to at most $2^{\circ}$ for the present data. This is essentially why $S_{y x}$ is expected to be zero. In fact, in the $\mathrm{FQH}$ regime, except in the narrow field regions where the fractions develop, we observe a constant ratio $S_{y x} / S_{x x} \approx-\epsilon_{x x} / \epsilon_{y x} \approx 0.35$, nearly independent of magnetic field and temperature (see insert in Fig. 2c; the slight increase at higher fields is probably due to a decrease of the Hall angle). The effective electric field is thus turned by an angle of about $20^{\circ}$ with respect to the temperature gradient. This effect may be due to the phonon anisotropy in the GaAs substrate which has already been observed at zero field [10].

Finally, the TEP is seen to change dramatically when passing through the fractional minimum at $\nu=1 / 3$, especially at temperatures below $1 \mathrm{~K}$. The TEP increases roughly by a factor of two from above $\nu=1 / 3$ to below $\nu=1 / 3$. At lower temperatures, and particularly in samples other than presented in Fig. 2, the TEP has actually been shown to be nearly field independent for $2 / 3<\nu<1 / 3$, as well as for $\nu<1 / 3$ [7]. In this previous paper it was concluded that the electronic quasiparticle states are subjected to a different electron-phonon interaction below and above $1 / 3$. This might be due to a change in their cohesive energy [11] leading to a change in the thermodynamic properties of the quasiparticle system [12].

In the following we concentrate on the overall behaviour (i.e. outside the regions where the FQHE minima develop) of the TEP in the quantum limit as a function of field and temperature. Although we present data mainly for the lowest concentration sample G647, we emphasize that all the main features were also observed in the other samples. Indeed from the somewhat limited number of samples that we have examined, we tentatively conclude that the behaviour of $S_{x x}$ at high fields appears to be universal.

An obvious feature is that $S_{x x}$ (Fig. 2a) is remarkably high, reaching values of up to 70 $\mathrm{mV} / \mathrm{K}$ for the presented data of sample G647 at a filling factor of about $\nu \approx 0.2$, much higher than expected by conventional theory [13].
Although we cannot yet explain the absolute values of the TEP in the FQH regime, we attempt to describe its general variation using a recent theory which calculates the phonon-drag induced current in an ideal 2DEG [3]. Since for high mobility samples $\rho_{y x}$ is accurately given by $\rho_{y x}=B / n e \propto B$ ( $n$ is the electron concentration), $S_{x x} \approx-\epsilon_{y x} \rho_{y x}$ reflects the properties of the fundamental thermoelectric quantity $\epsilon_{y x}$, i.e. of the phonon-drag induced current perpendicular to the thermal gradient, and the theoretically predicted drag current can be directly compared with our experimental TEP data.

The relevant temperature scale for the phonon drag TEP in a magnetic field is defined by the ratio between the thermal energy $k T$ and the characteristic energy $\hbar v / l_{\mathrm{B}}$ of the phonons which couple to the 2DEG ( $l_{\mathrm{B}}$ is the magnetic length and $v$ the phonon velocity), giving a characteristic temperature $T^{*}=\hbar v / k l_{\mathrm{B}}$. At fixed magnetic field and for very low temperatures $\left(T \ll T^{*}\right)$, the drag current $j_{\text {drag }}$ is predicted to be proportional to $T^{5}$ [3]. In the intermediate temperature range of $0.05<T / T^{*}<0.4, j_{\mathrm{drag}}$ varies approximately linearly with temperature. Finally, for high temperatures $\left(T \geq T^{*}\right)$ it saturates at a value $j_{\max }$. This dependence $f\left(k T l_{\mathrm{B}} / \hbar v\right)$ of $j_{\text {drag }}$ is represented in Fig. 4 for an arbitrarily chosen saturation value $j_{\max }$. Comparing the predicted temper-

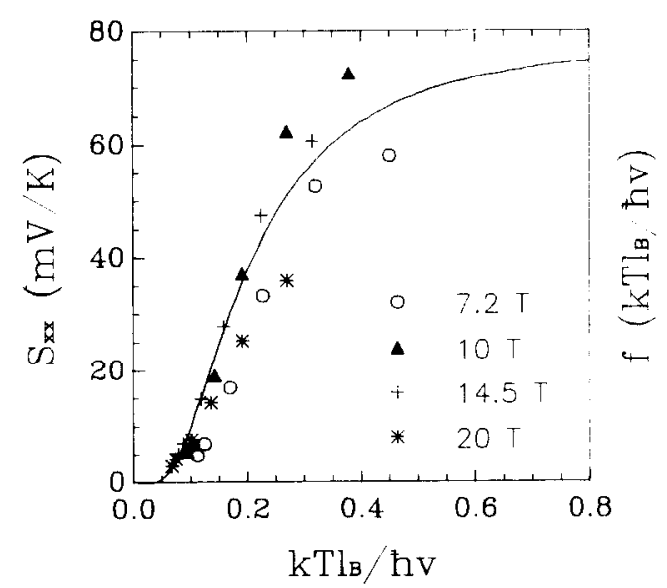

Fig. 4. The temperature dependence of $S_{x x}$ of G647 at different magnetic fields compared to the theoretically ex pected phonon-drag current. 
ature dependence of $j_{\text {drag }}$ with the experimentally measured values of $S_{x x}$ (we do not pretend to explain in detail the field dependence of $S_{x x} \propto$ $j_{\text {drag }} B$ ), we get good agreement between the experimental and theoretical variations even in the high field range $(\nu<0.2, B>10 \mathrm{~T})$, where the $B$ dependence of the TEP is not reproduced by the theory (see below).

The magnetic field dependence of the phonon drag current is predicted to be given by [3]:

$j_{\text {drag }} \propto \nu(1-\nu) l_{\mathrm{B}}^{-1} f\left(k T l_{\mathrm{B}} / \hbar v\right)(1+\delta)$

( $\nu \propto 1 / B$ is the filling factor, $\delta$ depends on the ratio between deformation potential and polar interaction). Using $S_{x x} \propto j_{\mathrm{drag}} B$, being in a range where $j_{\text {drag }}$ varies linearly with $k T l_{\mathrm{B}} / h v$, and supposing only a polar interaction $(\delta=0)$, the magnetic field dependence of the TEP will then be $S_{x x} \propto(1-\nu)$. We have plotted schematically the corresponding curve in Fig. 2a (dotted line). For higher fields $\left(l_{\mathrm{B}}<100 \AA\right)$, the deformation potential interaction becomes dominant, and the theoretical variation including this interaction is also schematically represented by the broken line in Fig. 2a. Up to filling factors of $\nu \approx 1 / 4$ the theoretical and experimental variations agree well, i.e., the TEP begins to rise rapidly on entering in the $\mathrm{FQH}$ regime and tends to level off at filling factors below $1 / 2$. However, at filling factors lower than $\nu \approx 0.2$, the experimental TEP begins to decrease again; there is, to our knowledge, no current theory which can explain this behaviour.

In summary, the low temperature TEP of $2 \mathrm{DEG}$ in three different $\mathrm{GaAs} / \mathrm{Ga}_{1-x} \mathrm{Al}_{x} \mathrm{As}$ heterojunctions has been measured in high magnetic fields at temperatures below $1 \mathrm{~K}$. We have shown that the TEP is due to phonon-drag and therefore reflects the nature of the phonon coupling to the electronic states. The general be- haviour was found to be in good agreement with recent theoretical calculations. However, numerous features are still not fully understood.

Part of this work was supported by NATO and by the National Science and Engineering Research Council of Canada. U.Z. would like to thank V.I. Fa'lko (MPI-FKF, Stuttgart) for stimulating discussions.

\section{References}

[1] R. Fletcher, J.C. Maan, and G. Weimann, Phys. Rev. B 32 (1985) 8477;

R. Fletcher, J.C. Maan, K. Ploog and G. Weimann, Phys. Rev. B 33 (1986) 7122;

R. Fletcher, M. D'lorio, W.T. Moore and R. Stoner, J. Phys. C: Solid State Phys. 21 (1988) 2681.

[2] H. Obloh, K. von Klitzing, K. Ploog and G. Weimann, Surf. Sci. 170 (1986) 292.

[3] V.I. Fal'ko and S.V. Iordanskii, J. Phys.: Condensed Matter 4 (1992) 9201.

[4] V.C. Karavolas and P.N. Butcher, J. Phys.: Condensed Matter 3 (1991) 2597.

[5] M.J, Smith and P.N. Butcher, J. Phys: Condensed Matter 2 (1990) 2375.

[6] G.M. Gusev, N.V. Zavaritskĩ, Z.D. Kvon and A.A. Yurgens, Pis'ma Zh. Eksp. Teor. Fiz. 40 (1984) 275, (JETP Lett. 40 (1984) 1056);

B.L. Gallagher, J.P. Oxley, T. Galloway, M.J. Smith and P.N. Butcher, J. Phys.: Condensed Matter 2 (1990) 755.

[7] U. Zeitler, R. Fletcher, J.C. Maan, C.T. Foxon, J.J. Harris and P. Wyder, Phys. Rev. B 47 (1993) 16008.

[8] S.S. Kubakaddi, P.N. Butcher and B.G. Muliamani, Phys. Rev. B 40 (1989) 1377; S.K. Lyo, Phys. Rev. B 40 (1989) 6458 .

[9] V.I. Fal'ko, S.V. Meshkov and S.V. Iordanskii, Phys. Rev. B 47 (1993) 9911 .

[10] H. Karl, W. Dietsche, A. Fischer and K. Ploog, Phys. Rev. Lett. 61 (1988) 2360.

[11] B.I. Halperin, Phys. Rev. Lett. 52 (1984) 1583, 2390(E).

[12] A.H. MacDonald, H.C.A. Oji and K.L. Liu, Phys. Rev. B 34 (1986) 2681.

[13] N.S. Sankeshwar, S.S. Kubakaddi, B.G. Mulimani and P.N. Butcher, J. Appl. Phys. 68 (1990) 5919. 